\title{
LIPSCHITZ AND ASYMPTOTIC STABILITY FOR PERTURBED NONLINEAR DIFFERENTIAL SYSTEMS
}

\author{
Yoon Hoe Goo
}

\begin{abstract}
The present paper is concerned with the notions of Lipschitz and asymptotic stability for perturbed nonlinear differential system knowing the corresponding stability of nonlinear differential system. We investigate Lipschitz and asymtotic stability for perturbed nonlinear differential systems. The main tool used is integral inequalities of the Bihari-type, in special some consequences of an extension of Bihari's result to Pinto and Pachpatte, and all that sort of things.
\end{abstract}

\section{INTRODUCTION}

The notion of uniformly Lipschitz stability (ULS) was introduced by Dannan and Elaydi [8] . For linear systems, the notions of uniformly Lipschitz stability and that of uniformly stability are equivalent. However, for nonlinear systems, the two notions are quite distinct. In fact, uniformly Lipschitz stability lies somewhere between uniformly stability on one side and the notions of asmptotic stability in variation of Brauer[4] and uniformly stability in variation of Brauer and Strauss[3] on the other side. Gonzalez and Pinto[9] proved theorems which relate the asymptotic behavior and boundedness of the solutions of nonlinear differential systems.

In this paper, we investigate Lipschitz and asymptotic stability for solutions of the nonlinear differential systems. To do this we need some integral inequalities. The method incorporating integral inequalities takes an important place among the methods developed for the qualitative analysis of solutions to linear and nonlinear system of differential equations. In the presence the method of integral inequalities is as efficient as the direct Lyapunov's method.

Received by the editors July 18, 2013. Revised October 23, 2013. Accepted November 25, 2013 2010 Mathematics Subject Classification. 34D10.

Key words and phrases. uniformly Lipschitz stability, uniformly Lipschitz stability in variation, exponentially asymptotic stability, exponentially asymptotic stability in variation. 


\section{Preliminaries}

We consider the nonlinear nonautonomous differential system

$$
x^{\prime}(t)=f(t, x(t)), \quad x\left(t_{0}\right)=x_{0},
$$

where $f \in C\left(\mathbb{R}^{+} \times \mathbb{R}^{n}, \mathbb{R}^{n}\right), \mathbb{R}^{+}=[0, \infty)$ and $\mathbb{R}^{n}$ is the Euclidean $n$-space. We assume that the Jacobian matrix $f_{x}=\partial f / \partial x$ exists and is continuous on $\mathbb{R}^{+} \times \mathbb{R}^{n}$ and $f(t, 0)=0$. Also, consider the perturbed differential system of $(2.1)$

$$
y^{\prime}=f(t, y)+\int_{t_{0}}^{t} g(s, y(s)) d s, y\left(t_{0}\right)=y_{0},
$$

where $g \in C\left(\mathbb{R}^{+} \times \mathbb{R}^{n}, \mathbb{R}^{n}\right), g(t, 0)=0$. For $x \in \mathbb{R}^{n}$, let $|x|=\left(\sum_{j=1}^{n} x_{j}^{2}\right)^{1 / 2}$. For an $n \times n$ matrix $A$, define the norm $|A|$ of $A$ by $|A|=\sup _{|x| \leq 1}|A x|$.

Let $x\left(t, t_{0}, x_{0}\right)$ denote the unique solution of $(2.1)$ with $x\left(t_{0}, t_{0}, x_{0}\right)=x_{0}$, existing on $\left[t_{0}, \infty\right)$. Then we can consider the associated variational systems around the zero solution of (2.1) and around $x(t)$, respectively,

$$
v^{\prime}(t)=f_{x}(t, 0) v(t), v\left(t_{0}\right)=v_{0}
$$

and

$$
z^{\prime}(t)=f_{x}\left(t, x\left(t, t_{0}, x_{0}\right)\right) z(t), z\left(t_{0}\right)=z_{0} .
$$

The fundamental matrix $\Phi\left(t, t_{0}, x_{0}\right)$ of $(2.4)$ is given by

$$
\Phi\left(t, t_{0}, x_{0}\right)=\frac{\partial}{\partial x_{0}} x\left(t, t_{0}, x_{0}\right),
$$

and $\Phi\left(t, t_{0}, 0\right)$ is the fundamental matrix of $(2.3)$.

Before giving further details, we give some of the main definitions that we need in the sequel[8].

Definition 2.1. The system (2.1) (the zero solution $x=0$ of (2.1)) is called

(S) stable if for any $\epsilon>0$ and $t_{0} \geq 0$, there exists $\delta=\delta\left(t_{0}, \epsilon\right)>0$ such that if $\left|x_{0}\right|<\delta$, then $|x(t)|<\epsilon$ for all $t \geq t_{0} \geq 0$,

(US) uniformly stable if the $\delta$ in (S) is independent of the time $t_{0}$,

(ULS) uniformly Lipschitz stable if there exist $M>0$ and $\delta>0$ such that $|x(t)| \leq$ $M\left|x_{0}\right|$ whenever $\left|x_{0}\right| \leq \delta$ and $t \geq t_{0} \geq 0$

(ULSV) uniformly Lipschitz stable in variation if there exist $M>0$ and $\delta>0$ such that $\left|\Phi\left(t, t_{0}, x_{0}\right)\right| \leq M$ for $\left|x_{0}\right| \leq \delta$ and $t \geq t_{0} \geq 0$,

(EAS) exponentially asymptotically stable if there exist constants $K>0, c>0$, 
and $\delta>0$ such that

$$
|x(t)| \leq K\left|x_{0}\right| e^{-c\left(t-t_{0}\right)}, 0 \leq t_{0} \leq t
$$

provided that $\left|x_{0}\right|<\delta$,

(EASV) exponentially asymptotically stable in variation if there exist constants $K>$ 0 and $c>0$ such that

$$
\left|\Phi\left(t, t_{0}, x_{0}\right)\right| \leq K e^{-c\left(t-t_{0}\right)}, 0 \leq t_{0} \leq t
$$

provided that $\left|x_{0}\right|<\infty$.

We give some related properties that we need in the sequel.

We need Alekseev formula to compare between the solutions of (2.1) and the solutions of perturbed nonlinear system

$$
y^{\prime}=f(t, y)+g(t, y), y\left(t_{0}\right)=y_{0},
$$

where $g \in C\left(\mathbb{R}^{+} \times \mathbb{R}^{n}, \mathbb{R}^{n}\right)$ and $g(t, 0)=0$. Let $y(t)=y\left(t, t_{0}, y_{0}\right)$ denote the solution of (2.5) passing through the point $\left(t_{0}, y_{0}\right)$ in $\mathbb{R}^{+} \times \mathbb{R}^{n}$.

The following is a generalization to nonlinear system of the variation of constants formula due to Alekseev [1].

Lemma 2.2. Let $x$ and $y$ be a solution of (2.1) and (2.5), respectively. If $y_{0} \in \mathbb{R}^{n}$, then for all $t$ such that $x\left(t, t_{0}, y_{0}\right) \in \mathbb{R}^{n}$,

$$
y\left(t, t_{0}, y_{0}\right)=x\left(t, t_{0}, y_{0}\right)+\int_{t_{0}}^{t} \Phi(t, s, y(s)) g(s, y(s)) d s .
$$

Lemma $2.3([7])$. Let $u, \lambda_{1}, \lambda_{2}, w \in C\left(\mathbb{R}^{+}\right), w(u)$ be nondecreasing in $u$ and $\frac{1}{v} w(u) \leq$ $w\left(\frac{u}{v}\right)$ for some $v>0$. If , for some $c>0$,

$$
u(t) \leq c+\int_{t_{0}}^{t} \lambda_{1}(s) u(s) d s+\int_{t_{0}}^{t} \lambda_{1}(s)\left\{\int_{t_{0}}^{s} \lambda_{2}(\tau) w(u(\tau)) d \tau\right\} d s, \quad t \geq t_{0} \geq 0
$$

then

$$
u(t) \leq W^{-1}\left[W(c)+\int_{t_{0}}^{t} \lambda_{2}(s) d s\right] \exp \left(\int_{t_{0}}^{t} \lambda_{1}(s) d s\right), t_{0} \leq t<b_{1},
$$

where $W(u)=\int_{u_{0}}^{u} \frac{d s}{w(s)}, u>0, u_{0}>0, W^{-1}(u)$ is the inverse of $W(u)$ and

$$
b_{1}=\sup \left\{t \geq t_{0}: W(c)+\int_{t_{0}}^{t} \lambda_{2}(s) d s \in \operatorname{dom}^{-1}\right\} .
$$


Lemma $2.4([10])$. Let $u, p, q, w$, and $r \in C\left(\mathbb{R}^{+}\right)$and suppose that, for some $c \geq 0$, we have

$$
u(t) \leq c+\int_{t_{0}}^{t} p(s) \int_{t_{0}}^{s}\left[q(\tau) u(\tau)+w(\tau) \int_{t_{0}}^{\tau} r(a) u(a) d a\right] d \tau d s, t \geq t_{0} .
$$

Then

$$
u(t) \leq c \exp \left(\int_{t_{0}}^{t} p(s) \int_{t_{0}}^{s}\left[q(\tau)+w(\tau) \int_{t_{0}}^{\tau} r(a) d a\right] d \tau d s\right), t \geq t_{0} .
$$

Lemma $2.5([15])$. Let $u(t), f(t)$, and $g(t)$ be real-valued nonnegative continuous functions defined on $\mathbb{R}^{+}$, for which the inequality

$$
u(t) \leq u_{0}+\int_{0}^{t} f(s) u(s) d s+\int_{0}^{t} f(s)\left(\int_{0}^{s} g(\tau) u(\tau) d \tau\right) d s, t \in \mathbb{R}^{+},
$$

holds, where $u_{0}$ is a nonnegative constant. Then,

$$
u(t) \leq u_{0}\left(1+\int_{0}^{t} f(s) \exp \left(\int_{0}^{s}(f(\tau)+g(\tau)) d \tau\right) d s\right), t \in \mathbb{R}^{+} .
$$

Lemma 2.6 ([12]). Let $u, \lambda_{1}, \lambda_{2}, \lambda_{3} \in C\left(\mathbb{R}^{+}\right), w \in C((0, \infty))$ and $w(u)$ be nondecreasing in $u, u \leq w(u)$. Suppose that for some $c>0$,

$$
u(t) \leq c+\int_{t_{0}}^{t} \lambda_{1}(s) w(u(s)) d s+\int_{t_{0}}^{t} \lambda_{2}(s)\left(\int_{t_{0}}^{s} \lambda_{3}(\tau) u(\tau) d \tau\right) d s, \quad 0 \leq t_{0} \leq t .
$$

Then

$$
u(t) \leq W^{-1}\left[W(c)+\int_{t_{0}}^{t}\left(\lambda_{1}(s)+\lambda_{2}(s) \int_{t_{0}}^{s} \lambda_{3}(\tau)\right) d s\right], t_{0} \leq t<b_{1},
$$

where $W, W^{-1}$ are the same functions as in Lemma 2.3 and

$$
b_{1}=\sup \left\{t \geq t_{0}: W(c)+\int_{t_{0}}^{t}\left(\lambda_{1}(s)+\lambda_{2}(s) \int_{t_{0}}^{s} \lambda_{3}(\tau)\right) d s \in \operatorname{domW}^{-1}\right\} .
$$

Lemma $2.7([13])$. Let $u, p, q, w, r \in C\left(\mathbb{R}^{+}\right), w \in C((0, \infty))$ and $w(u)$ be nondecreasing in $u$. Suppose that for some $c \geq 0$,

$$
u(t) \leq c+\int_{t_{0}}^{t}\left(p(s) \int_{t_{0}}^{s}\left(q(\tau) w(u(\tau))+v(\tau) \int_{t_{0}}^{\tau} r(a) w(u(a)) d a\right) d \tau\right) d s, t \geq t_{0} .
$$

Then

(2.10) $u(t) \leq W^{-1}\left[W(c)+\int_{t_{0}}^{t}\left(p(s) \int_{t_{0}}^{s}\left(q(\tau)+v(\tau) \int_{t_{0}}^{\tau} r(a) d a\right) d \tau\right) d s\right], t_{0} \leq t<b_{1}$,

where $W(u)=\int_{u_{0}}^{u} \frac{d s}{w(s)}, W^{-1}(u)$ is the inverse of $W(u)$ and

$$
b_{1}=\sup \left\{t \geq t_{0}: W(c)+\int_{t_{0}}^{t}\left(p(s) \int_{t_{0}}^{s}\left(q(\tau)+v(\tau) \int_{t_{0}}^{\tau} r(a) d a\right) d \tau\right) d s \in \operatorname{domW}^{-1}\right\} .
$$


Lemma $2.8([14])$. Let the following condition hold for functions $u(t), v(t) \in C\left[\left[t_{0}, \infty\right), \mathbb{R}^{+}\right)$ and $k(t, u) \in C\left[\left[t_{0}, \infty\right) \times \mathbb{R}^{n}, \mathbb{R}^{+}\right)$:

$$
u(t)-\int_{t_{0}}^{t} k(s, u(s)) d s \leq v(t)-\int_{t_{0}}^{t} k(s, v(s)) d s,
$$

$t \geq t_{0}$ and $k(s, u)$ is strictly increasing in $u$ for each fixed $s \geq 0$. If $u\left(t_{0}\right)<v\left(t_{0}\right)$, then $u(t)<v(t), t \geq t_{0} \geq 0$.

Lemma $2.9([5])$. Let $u, \lambda_{1}, \lambda_{2}, \lambda_{3} \in C\left(\mathbb{R}^{+}\right), w \in C((0, \infty))$ and $w(u)$ be nondecreasing in $u$. Suppose that for some $c>0$,

$$
u(t) \leq c+\int_{t_{0}}^{t} \lambda_{1}(s) w(u(s)) d s+\int_{t_{0}}^{t} \lambda_{2}(s)\left(\int_{t_{0}}^{s} \lambda_{3}(\tau) w(u(\tau)) d \tau\right) d s, \quad 0 \leq t_{0} \leq t .
$$

Then

$$
u(t) \leq W^{-1}\left[W(c)+\int_{t_{0}}^{t}\left(\lambda_{1}(s)+\lambda_{2}(s) \int_{t_{0}}^{s} \lambda_{3}(\tau)\right) d s\right], t_{0} \leq t<b_{1},
$$

where $W(u)=\int_{u_{0}}^{u} \frac{d s}{w(s)}, u>0, u_{0}>0, W^{-1}(u)$ is the inverse of $W(u)$ and

$$
b_{1}=\sup \left\{t \geq t_{0}: W(c)+\int_{t_{0}}^{t}\left(\lambda_{1}(s)+\lambda_{2}(s) \int_{t_{0}}^{s} \lambda_{3}(\tau)\right) d s \in \operatorname{domW}^{-1}\right\} .
$$

\section{Main Results}

In this section, we investigate Lipschitz and asymptotic stability for solutions of the nonlinear perturbed differential systems.

Theorem 3.1. Assume that $x=0$ of (2.1) is ULS. Let the following condition hold for (2.2):

$$
\int_{t_{0}}^{t}|g(s, y(s))| d s \leq W(t,|y|), 0 \leq t_{0} \leq t
$$

where $W(t, u) \in C\left(\mathbb{R}^{+} \times \mathbb{R}^{+}, \mathbb{R}^{+}\right)$is monotone nondecreasing in u with $W(t, 0)=0$. Suppose that $u(t)$ is any solution of the scalar differential equation

$$
u^{\prime}(t)=M W(t, u), u\left(t_{0}\right)=u_{0}>0, M \geq 1,
$$

existing on $\mathbb{R}^{+}$such that $m\left(t_{0}\right)<u\left(t_{0}\right)$. If $u=0$ of (3.1) is ULS, then $y=0$ of (2.2) is also ULS whenever $M\left|y_{0}\right|<u_{0}$.

Proof. Let $x(t)=x\left(t, t_{0}, y_{0}\right)$ and $y(t)=y\left(t, t_{0}, y_{0}\right)$ be solutions of (2.1) and (2.2), respectively. Using the variation of constants formula, we have 


$$
|y(t)| \leq|x(t)|+\int_{t_{0}}^{t}|\Phi(t, s, y(s))| \int_{t_{0}}^{s}|g(\tau, y(\tau))| d \tau d s
$$

where $\Phi\left(t, t_{0}, y_{0}\right)$ is the fundemental matrix of (2.4). Since $x=0$ of (2.1) is ULS, it is ULSV by Corollary 3.6[5]. Thus there exist $M>0$ and $\delta>0$ such that $\left|\Phi\left(t, t_{0}, y_{0}\right)\right| \leq M$ for $t \geq t_{0} \geq 0$. Therefore, by the assmption, we have

$$
|y(t)|-M \int_{t_{0}}^{t} W(s,|y(s)|) d s \leq M\left|y_{0}\right|<u_{0}=u(t)-M \int_{t_{0}}^{t} W(s, u(s)) d s .
$$

Hence $|y(t)|<u(t)$ by Lemma 2.8. Since $u=0$ of (3.1) is ULS, it easily follows that $y=0$ of $(2.2)$ is ULS.

Corollary 3.2. Assume that $x=0$ of (2.1) is ULS. Consider the scalar differential equation

$$
u^{\prime}(t)=K W(t, u)=K a(t)\left[u+\int_{t_{0}}^{t} k(s) u(s) d s\right],
$$

where $u_{0} \geq 1, K \geq 1$ and $a, k \in C\left(\mathbb{R}^{+}\right)$satisfy the conditions

(a) $\int_{t_{0}}^{t}|g(s, y(s))| d s \leq W(t,|y|)$, where $\int_{t_{0}}^{t} g(s, y(s)) d s$ is in (2.2),

(b) $M\left(t_{0}\right)=\left(1+K \int_{t_{0}}^{\infty} a(s) \exp \left(\int_{t_{0}}^{s}(K a(\tau)+k(\tau)) d \tau\right) d s\right)<\infty$ and $b_{1}=\infty$.

Then $y=0$ of (2.2) is ULS.

Proof. Let $u(t)=u\left(t, t_{0}, x_{0}\right)$ be any solution of (3.2). Then, by Lemma 2.5, we have

$$
|u(t)| \leq u_{0}\left(1+K \int_{t_{0}}^{t} a(s) \exp \left(\int_{t_{0}}^{s}(K a(\tau)+k(\tau)) d \tau\right) d s\right) \leq M\left(t_{0}\right)\left|u_{0}\right|
$$

Hence $u=0$ of (3.2) is ULS. This implies that the solution $y=0$ of (2.2) is ULS by Theorem 3.1.

Remark 3.3. In Corollary 3.2 , it is needed that $b_{1}=\infty$. The condition $W(\infty)=\infty$ is too strong and it represents situations which are not stable. For example, if $w(u)=u^{\alpha}$, then only $\alpha \leq 1$ satisfies $W(\infty)=\infty$ and $\alpha<1$ is not stable. See [18].

Corollary 3.4. Assume that $x=0$ of (2.1) is ULS. Consider the scalar differential equation

$$
u^{\prime}(t)=K W(t, u)=K a(t)\left[u+\int_{t_{0}}^{t} k(s) w(u(s)) d s\right],
$$

where $u_{0} \geq 1, K \geq 1, u, w \in C\left(\mathbb{R}^{+}\right), w(u)$ be nondecreasing in $u$ and $\frac{1}{v} w(u) \leq w\left(\frac{u}{v}\right)$ for some $v>0$, and $a, k \in C\left(\mathbb{R}^{+}\right)$satisfy the conditions

(a) $\int_{t_{0}}^{t}|g(s, y(s))| d s \leq W(t,|y|)$, where $\int_{t_{0}}^{t} g(s, y(s)) d s$ is in (2.2), 
(b) $M\left(t_{0}\right)=W^{-1}\left[W\left(u_{0}\right)+\int_{t_{0}}^{\infty} k(s) d s\right] \cdot \exp \left(\int_{t_{0}}^{\infty} K a(s) d s\right)<\infty, b_{1}=\infty$, and $a, k \in$ $L_{1}\left(\mathbb{R}^{+}\right)$. Then $y=0$ of (2.2) is ULS.

Proof. Let $u(t)=u\left(t, t_{0}, x_{0}\right)$ be any solution of (3.3). Then, by Lemma 2.3, we have

$$
|u(t)| \leq W^{-1}\left[W\left(u_{0}\right)+\int_{t_{0}}^{t} k(s) d s\right] \cdot \exp \left(\int_{t_{0}}^{t} K a(s) d s\right) \leq M\left(t_{0}\right) \leq M\left(t_{0}\right)\left|u_{0}\right| .
$$

Hence $u=0$ of (3.3) is ULS. By Theorem 3.1, the solution $y=0$ of (2.2) is ULS.

Corollary 3.5. Assume that $x=0$ of (2.1) is ULS. Consider the scalar differential equation

$$
u^{\prime}(t)=K W(t, u)=K\left[a(t) w(u(t))+b(s) \int_{t_{0}}^{t} k(s) u(s) d s\right]
$$

where $w \in C\left((0, \infty), w(u)\right.$ is nondecreasing on $u$ and $u \leq w(u), u_{0} \geq 1, K \geq 1$ and $a, b, k \in C\left(\mathbb{R}^{+}\right)$satisfy the conditions

(a) $\int_{t_{0}}^{t}|g(s, y(s))| d s \leq W(t,|y|)$, where $\int_{t_{0}}^{t} g(s, y(s)) d s$ is in (2.2),

(b) $M\left(t_{0}\right)=W^{-1}\left[W\left(u_{0}\right)+K \int_{t_{0}}^{\infty}\left(a(s)+b(s) \int_{t_{0}}^{s} k(s) d s\right)\right]<\infty, b_{1}=\infty$, and $a, b, k \in$ $L_{1}\left(\mathbb{R}^{+}\right)$. Then $y=0$ of (2.2) is $U L S$.

Proof. Let $u(t)=u\left(t, t_{0}, x_{0}\right)$ be any solution of (3.4). Then, Lemma 2.6, we have

$$
|u(t)| \leq W^{-1}\left[W\left(u_{0}\right)+K \int_{t_{0}}^{t}\left(a(s)+b(s) \int_{t_{0}}^{s} k(s) d s\right)\right] \leq M\left(t_{0}\right) \leq M\left(t_{0}\right)\left|u_{0}\right| .
$$

Hence $u=0$ of (3.4) is ULS, and so by Theorem 3.1, the solution $y=0$ of $(2.2)$ is ULS.

Theorem 3.6. For the perturbed (2.2), we asssume that

$$
\int_{t_{0}}^{t}|g(s, y(s))| d s \leq a(t) w(|y(t)|)+b(t) \int_{t_{0}}^{t} k(s)|y(s)| d s,
$$

where $a, b, k \in C\left(\mathbb{R}^{+}\right), a, b, k \in L_{1}\left(\mathbb{R}^{+}\right), w \in C((0, \infty)$, and $w(u)$ is nondecreasing in $u, u \leq w(u)$, and $\frac{1}{v} w(u) \leq w\left(\frac{u}{v}\right)$ for some $v>0$,

$$
M\left(t_{0}\right)=W^{-1}\left[W(M)+M \int_{t_{0}}^{\infty}\left(a(s)+b(s) \int_{t_{0}}^{s} k(\tau) d \tau\right) d s\right]
$$

where $M\left(t_{0}\right)<\infty$ and $b_{1}=\infty$. Then the zero solution of (2.2) is ULS whenever the zero solution of (2.1) is ULSV.

Proof. Let $x(t)=x\left(t, t_{0}, y_{0}\right)$ and $y(t)=y\left(t, t_{0}, y_{0}\right)$ be solutions of (2.1) and (2.2), respectively. Since $x=0$ of (2.1) is ULSV, it is ULS by Theorem 3.3[8]. Applying 
Lemma 2.2, we have

$$
\begin{aligned}
|y(t)| \leq & |x(t)|+\int_{t_{0}}^{t}|\Phi(t, s, y(s))|\left|\int_{t_{0}}^{s} g(\tau, y(\tau)) d \tau\right| d s \\
\leq & M\left|y_{0}\right|+\int_{t_{0}}^{t} M\left|y_{0}\right| a(s) w\left(\frac{|y(s)|}{\left|y_{0}\right|}\right) d s \\
& +\int_{t_{0}}^{t} M\left|y_{0}\right| b(s) \int_{t_{0}}^{s} k(\tau) \frac{|y(\tau)|}{\left|y_{0}\right|} d \tau d s .
\end{aligned}
$$

Set $u(t)=|y(t)|\left|y_{0}\right|^{-1}$. Now an application of Lemma 2.6 yields

$$
|y(t)| \leq\left|y_{0}\right| W^{-1}\left[W(M)+M \int_{t_{0}}^{t}\left(a(s)+b(s) \int_{t_{0}}^{s} k(\tau) d \tau\right) d s\right] .
$$

Hence we have $|y(t)| \leq M\left(t_{0}\right)\left|y_{0}\right|$ for some $M\left(t_{0}\right)>0$ whenever $\left|y_{0}\right|<\delta$. This completes the proof.

Theorem 3.7. For the perturbed (2.2), we asssume that

$$
|g(t, y)| \leq a(t) w(|y(t)|)+b(t) \int_{t_{0}}^{t} k(s)|y(s)| d s,
$$

where $a, b, k \in C\left(\mathbb{R}^{+}\right), a, b, k \in L_{1}\left(\mathbb{R}^{+}\right), w \in C((0, \infty)$, and $w(u)$ is nondecreasing in $u, u \leq w(u)$, and $\frac{1}{v} w(u) \leq w\left(\frac{u}{v}\right)$ for some $v>0$,

$$
M\left(t_{0}\right)=W^{-1}\left[W(M)+M \int_{t_{0}}^{\infty} \int_{t_{0}}^{s}\left(a(\tau)+b(\tau) \int_{t_{0}}^{\tau} k(r) d r\right) d \tau d s\right]
$$

where $M\left(t_{0}\right)<\infty$ and $b_{1}=\infty$. Then the zero solution of (2.2) is ULS whenever the zero solution of (2.1) is ULSV.

Proof. Let $x(t)=x\left(t, t_{0}, y_{0}\right)$ and $y(t)=y\left(t, t_{0}, y_{0}\right)$ be solutions of $(2.1)$ and (2.2), respectively. Using the nonlinear variation of constants formula and the ULSV condition of $x=0$ of $(2.1)$, we have

$$
\begin{aligned}
|y(t)| \leq & |x(t)|+\int_{t_{0}}^{t}|\Phi(t, s, y(s))| \int_{t_{0}}^{s}|g(\tau, y(\tau))| d \tau d s \\
\leq & M\left|y_{0}\right|+\int_{t_{0}}^{t} M\left|y_{0}\right| \int_{t_{0}}^{s}\left[a(\tau) w\left(\frac{|y(\tau)|}{\left|y_{0}\right|}\right) d \tau d s\right. \\
& \left.+\int_{t_{0}}^{t} M\left|y_{0}\right| \int_{t_{0}}^{s} b(\tau) \int_{t_{0}}^{\tau} k(r) \frac{|y(r)|}{\left|y_{0}\right|} d r\right] d \tau d s .
\end{aligned}
$$

Set $u(t)=|y(t)|\left|y_{0}\right|^{-1}$. Now an application of Lemma 2.7 yields

$$
|y(t)| \leq\left|y_{0}\right| W^{-1}\left[W(M)+M \int_{t_{0}}^{t} \int_{t_{0}}^{s}\left(a(\tau)+b(\tau) \int_{t_{0}}^{\tau} k(r) d r\right) d \tau d s\right],
$$


Thus we have $|y(t)| \leq M\left(t_{0}\right)\left|y_{0}\right|$ for some $M\left(t_{0}\right)>0$ whenever $\left|y_{0}\right|<\delta$, and so the proof is complete.

Theorem 3.8. Let the solution $x=0$ of (2.1) be EAS. Suppose that the perturbing term $g(t, y)$ satisfies

$$
|g(t, y(t))| \leq e^{-\alpha t}\left(a(t)|y(t)|+b(t) \int_{t_{0}}^{t} k(s)|y(s)| d s\right),
$$

where $\alpha>0, a, b, k \in C\left(\mathbb{R}^{+}\right), a, b, k \in L_{1}\left(\mathbb{R}^{+}\right), w(u)$ is nondecreasing in $u$, and $\frac{1}{v} w(u) \leq w\left(\frac{u}{v}\right)$ for some $v>0$. If

$$
M\left(t_{0}\right)=c \exp \left(\int_{t_{0}}^{\infty} M e^{\alpha s} \int_{t_{0}}^{s}\left[a(\tau)+b(\tau) \int_{t_{0}}^{\tau} k(r) d r\right] d \tau d s\right)<\infty, t \geq t_{0},
$$

where $c=\left|y_{0}\right| M e^{\alpha t_{0}}$, then all solutions of (2.2) approch zero as $t \rightarrow \infty$

Proof. Let $x(t)=x\left(t, t_{0}, y_{0}\right)$ and $y(t)=y\left(t, t_{0}, y_{0}\right)$ be solutions of (2.1) and (2.2), respectively. Since the solution $x=0$ of $(2.1)$ is $\mathrm{EAS}$, we have $\left|\Phi\left(t, t_{0}, x_{0}\right)\right| \leq$ $M e^{-\alpha\left(t-t_{0}\right)}$ for some $M>0$ and $c>0$ (Theorem 2[2]). Using Lemma 2.2, we have

$$
\begin{aligned}
|y(t)| \leq & |x(t)|+\int_{t_{0}}^{t}|\Phi(t, s, y(s))|\left|\int_{t_{0}}^{s} g(\tau, y(\tau)) d \tau\right| d s \\
\leq & M\left|y_{0}\right| e^{-\alpha\left(t-t_{0}\right)}+\int_{t_{0}}^{t} M e^{-\alpha(t-s)} \int_{t_{0}}^{s}\left[a(\tau) e^{-\alpha \tau}|y(\tau)|\right. \\
& \left.+b(\tau) \int_{t_{0}}^{\tau} k(r) e^{-\alpha r}|y(r)| d r d \tau\right] d s,
\end{aligned}
$$

since $e^{\alpha t}$ is increasing. Set $u(t)=|y(t)| e^{\alpha t}$. An application of Lemma 2.4 obtains $|y(t)| \leq c e^{-\alpha t} \exp \left(\int_{t_{0}}^{t} M e^{\alpha s} \int_{t_{0}}^{s}\left[a(\tau)+b(\tau) \int_{t_{0}}^{\tau} k(r) d r\right] d \tau d s\right) \leq c e^{-\alpha t} M\left(t_{0}\right), t \geq t_{0}$.

The above estimation yields the desired result.

Theorem 3.9. Let the solution $x=0$ of (2.1) be EAS. Suppose that the perturbing term $g(t, y)$ satisfies

$$
\int_{t_{0}}^{t}|g(s, y(s))| d s \leq e^{-\alpha t}\left(a(t) w(|y(t)|)+b(t) \int_{t_{0}}^{t} k(s) w(|y(s)|) d s\right),
$$

where $\alpha>0, a, b, k, w \in C\left(\mathbb{R}^{+}\right), a, b, k \in L_{1}\left(\mathbb{R}^{+}\right)$and $w(u)$ is nondecreasing in $u$. If

$$
M\left(t_{0}\right)=W^{-1}\left[W(c)+M \int_{t_{0}}^{\infty}\left(a(s)+b(s) \int_{t_{0}}^{s} k(\tau) d \tau\right) d s\right]<\infty, b_{1}=\infty,
$$

where $c=M\left|y_{0}\right| e^{\alpha t_{0}}$, then all solutions of (2.2) approch zero as $t \rightarrow \infty$ 
Proof. Let $x(t)=x\left(t, t_{0}, y_{0}\right)$ and $y(t)=y\left(t, t_{0}, y_{0}\right)$ be solutions of (2.1) and (2.2), respectively. Using Lemma 2.2 and the assmptions, we have

$$
\begin{aligned}
|y(t)| \leq & |x(t)|+\int_{t_{0}}^{t}|\Phi(t, s, y(s))|\left|\int_{t_{0}}^{s} g(\tau, y(\tau)) d \tau\right| d s \\
\leq & M\left|y_{0}\right| e^{-\alpha\left(t-t_{0}\right)}+\int_{t_{0}}^{t} M e^{-\alpha(t-s)}\left[e^{-\alpha s} a(s) w(|y(s)|)\right. \\
& \left.+M b(s) e^{-\alpha s} \int_{t_{0}}^{s} k(\tau) w(|y(\tau)|) d \tau\right] d s .
\end{aligned}
$$

Set $u(t)=|y(t)| e^{\alpha t}$. Since $w(u)$ is nondecreasing, an application of Lemma 2.9 obtains

$$
|y(t)| \leq e^{-\alpha t} W^{-1}\left[W(c)+M \int_{t_{0}}^{t}\left(a(s)+b(s) \int_{t_{0}}^{s} k(\tau) d \tau\right) d s\right],
$$

where $c=M\left|y_{0}\right| e^{\alpha t_{0}}$. From the above estimation, we obtains the desired result.

Acknowledgement. The author is very grateful for the referee's valuable comments.

\section{REFERENCES}

1. V.M. Alekseev: An estimate for the perturbations of the solutions of ordinary differential equations. Vestn. Mosk. Univ. Ser. I. Math. Mekh.(Russian) 2 (1961), 28-36.

2. F. Brauer: Perturbations of nonlinear systems of differential equations, II. J. Math. Anal. Appl. 17 (1967), 418-434.

3. F. Brauer \& A. Strauss: Perturbations of nonlinear systems of differential equations, III. J. Math. Anal. Appl. 31 (1970), 37-48.

4. F. Brauer. Perturbations of nonlinear systems of differential equations, IV. J. Math. Anal. Appl. 37 (1972), 214-222.

5. S.K. Choi \& N.J. Koo: $h$-stability for nonlinear perturbed systems. Ann. Diff. Eqs. 11 (1995), 1-9.

6. S.K. Choi, Y.H. Goo \& N.J. Koo: Lipschitz and exponential asymptotic stability for nonlinear functional systems. Dynamic Systems and Applications 6 (1997), 397-410.

7. S.K. Choi, N.J. Koo \& S.M. Song: Lipschitz stability for nonlinear functional differential systems. Far East J. Math. Sci(FJMS)I 5 (1999), 689-708.

8. F.M. Dannan \& S. Elaydi: Lipschitz stability of nonlinear systems of differential systems. J. Math. Anal. Appl. 113 (1986), 562-577.

9. P. Gonzalez \& M. Pinto: Stability properties of the solutions of the nonlinear functional differential systems. J. Math. Anal. Appl. 181 (1994), 562-573. 
10. Y.H. Goo \& S.B. Yang: $h$-stability of the nonlinear perturbed differential systems via $t_{\infty}$-similarity. J. Chungcheong Math. Soc. 24 (2011), 695-702.

11. _ $: h$-stability of nonlinear perturbed differential systems via $t_{\infty}$-similarity. $J$. Korean Soc. Math. Educ. Ser. B: Pure Appl. Math. 19 (2012), 171-177.

12. Y.H. Goo: Boundedness in the perturbed differential systems. J. Korean Soc. Math. Educ. Ser. B: Pure Appl. Math. 20 (2013), 223-232.

13. _ B B B B Chundedness in perturbed nonlinear differential systems. J. Chen Math. Soc. 26 (2013), 605-613.

14. V. Lakshmikantham \& S. Leela: Differential and Integral Inequalities: Theory and Applications Vol.I. Academic Press, New York and London, 1969.

15. B.G. Pachpatte: A note on Gronwall-Bellman inequality. J. Math. Anal. Appl. 44 (1973), 758-762.

16. M. Pinto: Perturbations of asymptotically stable differential systems. Analysis 4 (1984), 161-175.

17. ___ Integral inequalities of Bihari-type and applications. Funkcial. Ekvac. 33 (1990), 387-404.

18. ___ Variationally stable differential system. J. Math. Anal. Appl. 151 (1990), 254260.

Department of Mathematics, Hanseo University, Seosan, Chungnam, 356-706, Republic OF KOREA

Email address: yhgoo@hanseo.ac.kr 